\title{
SITUACIÓN EPIDEMIOLÓGICA DEL COVID-19 EN SUDAMÉRICA
}

\author{
EPIDEMIOLOGICAL SITUATION OF COVID-19 IN SOUTH AMERICA
}

R. Rainer Echeverría', J. Harumi Sueyoshi'

\section{Sr. Editor}

Actualmente la infección por coronavirus (COVID-19) se ha convertido en un problema de salud pública a nivel mundial. En diciembre del 2019, en la ciudad de Wuhan, provincia de Hubei, China, se reportaron los primeros casos de neumonía de etiología desconocida, los cuales se incrementaron rápidamente en otras provincias del país(1).

Posteriormente, se identificó al coronavirus SARS-CoV-2 como agente causal y, a mediados de enero del 2020 la Organización Mundial de la Salud (OMS) reportó más de 280 casos confirmados de COVID-19 en China, Tailandia, Japón y Corea ${ }^{(2)}$.

En Sudamérica se da a conocer el primer caso de COVID-19, el 26 de febrero del 2020 en la ciudad de Sao Paulo, Brasil; identificándose un paciente varón de 61 años proveniente de la región de Lombardía, Italia. Seguidamente se confirmarían otros casos importados del continente europeo y asiático en los demás países sudamericanos ${ }^{(3)}$.

Hasta el 14 de abril del 2020, se han reportado a nivel mundial 1983219 casos de COVID-19, de los cuales el 2,84\% se encuentra en América del Sur. Brasil es el país que presenta el mayor número de infectados (24 232), concentrados principalmente en Sao Paulo y Río de Janeiro. Perú tiene el segundo lugar con 10 303 casos confirmados, duplicando el número de infectados en los últimos 5 días. Este incremento se ve afectado por el aumento de las pruebas realizadas y a la falta de apego de un grupo de la población a las medidas establecidas por el gobierno, ocasionando aglomeraciones en supermercados, mercados y en transporte público.

En el Perú, los varones representan el 73,9\% del total de casos confirmados para COVID-19, hallándose la mayor cantidad de infectados en la capital del país (Lima), tendencia que se repite en las demás capitales latinoamericanas; mientras que en Chile el $50,09 \%$ de los casos positivos corresponden al sexo femenino. Las comorbilidades identificadas con mayor frecuencia en los casos fatales fueron las enfermedades cardiovasculares (hipertensión arterial) y diabetes mellitus (Perú, Chile y Brasil)(4,5).

En cuanto a mortalidad por COVID-19 en Sudamérica, Ecuador lleva la delantera, presentando hasta el momento una tasa de mortalidad de 2 por 100 mil habitantes; seguido de Perú con 0,7 por 100 mil habitantes.

A pesar de que Chile es uno de los países en Sudamérica que concentra la mayor cantidad de infectados por el SARS-CoV2, tiene la tasa de letalidad más baja de la región (1,16\%), seguido de Uruguay, incluso menos que Japón (1,87\%). Esto se debe a las medidas que han tomado sus autoridades para frenar la propagación de la enfermedad la cual incluye la capacidad para identificar pacientes infectados y aislarlos de forma temprana, estrategia en la que ha hecho hincapié la Organización Mundial de la Salud; logrando realizar hasta el momento la mayor cantidad de pruebas por millón de habitantes en la región, después de Venezuela. Actualmente Perú ha adoptado esta medida con fuerza, siendo el segundo país con mayor número de pruebas diagnósticas en Latinoamérica, con 102216 entre pruebas rápidas y molecular (ver Tabla 1).

${ }^{1}$ Universidad Nacional Mayor de San Marcos, Lima-Perú.

Citar como: R. Rainer Echeverría, J. Harumi Sueyoshi. Situación epidemiológica del COVID-19 en Sudamérica. Rev. Fac. Med. Hum. Julio 2020; 20(3):525-527. DOI 10.25176/RFMH.v20i3.2945 
Tabla 1. Datos estadísticos sobre COVID-19 en Sudamérica, 2020.

\begin{tabular}{|c|c|c|c|c|c|c|c|}
\hline $\begin{array}{l}\text { Países de } \\
\text { América } \\
\text { del Sur }\end{array}$ & $\begin{array}{l}\text { Primer caso } \\
\text { reportado }\end{array}$ & $\begin{array}{l}\text { Inicio de } \\
\text { cuarentena }\end{array}$ & $\begin{array}{c}\text { Casos } \\
\text { confirmados }\end{array}$ & Defunciones & Letalidad & $\begin{array}{c}\text { Pruebas } \\
\text { total }\end{array}$ & $\begin{array}{c}\text { Pruebas / } \\
\text { Millón }\end{array}$ \\
\hline Argentina & 3 de marzo & 20 de marzo & 2,277 & 102 & $4,47 \%$ & 22,805 & 505 \\
\hline Bolivia & 10 de marzo & 22 de marzo & 354 & 28 & $7,90 \%$ & 2,185 & 187 \\
\hline Brasil & $\begin{array}{l}26 \text { de } \\
\text { febrero }\end{array}$ & $\begin{array}{c}\text { Cierre de } \\
\text { fronteras } 19 \\
\text { marzo }\end{array}$ & 24,232 & 1,378 & $5,68 \%$ & 62,985 & 296 \\
\hline Chile & 3 de marzo & $\begin{array}{l}\text { Estado de } \\
\text { excepción } 18 \\
\text { de marzo }\end{array}$ & 7,917 & 92 & $1,16 \%$ & 87,794 & 4,593 \\
\hline Colombia & 6 de marzo & 24 de marzo & 2,852 & 112 & $3,92 \%$ & 43,053 & 846 \\
\hline Ecuador & $\begin{array}{l}29 \text { de } \\
\text { febrero }\end{array}$ & $\begin{array}{l}\text { Estado de } \\
\text { excepción } 16 \\
\text { de marzo }\end{array}$ & 7,603 & 355 & $4,66 \%$ & 25,347 & 1,437 \\
\hline Paraguay & 7 de marzo & 10 de marzo & 159 & 7 & $4,40 \%$ & 3,394 & 476 \\
\hline Perú & 6 de marzo & 15 de marzo & 10,303 & 230 & $2,23 \%$ & 102,216 & 3,100 \\
\hline Uruguay & 13 de marzo & $\begin{array}{c}\text { No } \\
\text { cuarentena }\end{array}$ & 483 & 8 & $1,65 \%$ & 9,236 & 2,659 \\
\hline Venezuela & 13 de marzo & 17 de marzo & 189 & 9 & $4,76 \%$ & 203,108 & 7,143 \\
\hline Total & & & 56,369 & 2,321 & $4,11 \%$ & & \\
\hline
\end{tabular}

Fuente: Coronavirus Resource Center Johns Hopkins University y Worldometer coronavirus (Actualizado: 14 de abril del 2020)(6,7).

Así mismo, la tasa de letalidad refleja el nivel de desarrollo y calidad del sistema de salud de cada país. A pesar de que el gasto en salud según el porcentaje de producto bruto interno en Bolivia $(6,3 \%)$ no tiene mucha diferencia respecto a Chile $(7,7 \%)^{(8)}$, Bolivia tiene una tasa de letalidad por coronavirus de 7,9\%, la más alta de América del Sur. Este valor está influenciado por la menor cantidad de pruebas diagnósticas realizadas, el tiempo de inicio de la infección, las medidas adoptadas por cada sistema sanitario y por las características propias de cada país.

Con la información actual puede observarse que las cifras globales en América del Sur aún no llegan a niveles comparables con otras regiones como Asia o Europa, incluso ninguno parece haber alcanzado el nivel de inflexión en su curva epidemiológica. Es necesario tomar en cuenta que existe un subregistro en cuanto a los datos reportados por cada país, pues este depende de la cantidad de pruebas de tamizaje que se aplique en la población; además, se recalca que los portadores asintomáticos pueden no estar considerados dentro de las estadísticas, ya que solo se suele realizar pruebas en personas sintomáticas.

Otra clave para el éxito en el control de la propagación de la enfermedad es la responsabilidad y la disciplina de la población al momento de seguir las medidas de seguridad establecidas por cada gobierno, como se ha demostrado en Corea del Sur ${ }^{(9)}$.

La propagación del COVID-19 pone a prueba los sistemas de salud, planteando un verdadero reto al momento de establecer políticas sanitarias y reforzamiento de los mismos, los cuales ya presentaban deficiencias antes de la pandemia ${ }^{(10)}$. Una de las grandes carencias que presentan es la falta de equipos de protección personal, la disponibilidad de camas en la unidad de cuidados intensivos y ventiladores mecánicos, piedra angular para el manejo de pacientes con infección por SARS-CoV- $2^{(3)}$.

Resulta complicado intentar comparar las cifras entre naciones e incluso las medidas adoptadas por cada uno de ellos, pues existen variables demográficas, económicas y políticas que pueden intervenir, así como también influye la cantidad de pruebas aplicadas, la densidad poblacional y el contexto social que vive cada país, factores que convierten a Sudamérica como un blanco vulnerable ${ }^{(11)}$.

Aún es necesario realizar más estudios para aumentar el bagaje sobre el conocimiento actual. 
Contribuciones de autoría: Los autores realizaron la generación, recolección de información, redacción y versión final del artículo original.

Financiamiento: Autofinanciado.

Correspondencia: Echeverría lbazeta, R. Rainer.

Dirección: H.H. Buenos Aires 1713 Bellavista, Callao-Perú.

Teléfono: +51970094986

Correo: r.rainer.echeverria@gmail.com

\section{REFERENCIAS BIBLIOGRÁFICAS}

1. Zhang G., Zhang J., Wang B., et al. Analysis of clinical characteristics and laboratory findings of 95 cases of 2019 novel coronavirus pneumonia in Wuhan, China: a retrospective analysis. Respir Res 21, 74. 2020. DOI: https:// doi.org/10.1186/s12931-020-01338-8.

2. Novel Coronavirus (2019-nCoV) SITUATION REPORT - 1. WHO. 2020 Disponible en: https://www.who.int/docs/default-source/coronaviruse/ situation-reports/20200121-sitrep-1-2019-ncov.pdf?sfvrsn=20a99c10_4.

3. Rodriguez A., Gallego V., Escalera J., Cimerman S., et al. COVID-19 in Latin America: The implications of the first confirmed case in Brazil, Travel Med Infect Dis. 2020. DOI: 10.1016/j.tmaid.2020.101613.

4. Sala situacional Covid-19, Ministerio de Salud - Perú. 2020 Disponible en: https://covid19.minsa.gob.pe/sala_situacional.asp

5. Séptimo informe epidemiológico COVID-19. Ministerio de salud de Chile Plan de acción coronavirus. 2020. Disponible en: https://www.minsal.cl/ nuevo-coronavirus-2019-ncov/informe-epidemiologico-covid-19/

6. Resource center Johns Hopkins University. Disponible en: https:// coronavirus.jhu.edu/map.html
Conflicto de interés: Los autores declaran no tener conflictos de interés en la publicación de este artículo.

Recibido: 15 de abril 2020

Aprobado: 22 de mayo 2020
7. Worldometer coronavirus. Disponible en: https://www.worldometers. info/coronavirus/

8. Salud en las Américas. OMS/OPS.2017 №642. Disponible en: https:// www.paho.org/salud-en-las-americas-2017/wp-content/uploads/2017/09/ Print-Version-Spanish.pdf

9. Shim E., Tariq A., Wongyeong C., Lee Y., Chowell G., Transmission potential of COVID-19 in South Korea. Int Journal of Infect Dis. 2020. 399-344. DOI: 10.1016/j.ijid.2020.03.031

10. Pérez J., La necesaria reforma de los Sistemas de Salud en América Latina. Gaceta Laboral. 2007,13(1) 43-57. Disponible en: http://ve.scielo.org/ scielo.php?script=sci_arttext\&pid=\$1315-85972007000100003

11. Rifat A., Monteiro L., Almeida G., et al. Health-system reform and universal heath coverage in Latin America. The Lancet, Volume 385, Issue 9974, 1230 - 1247. DOI: 10.1016/S0140-6736(14)61646-9

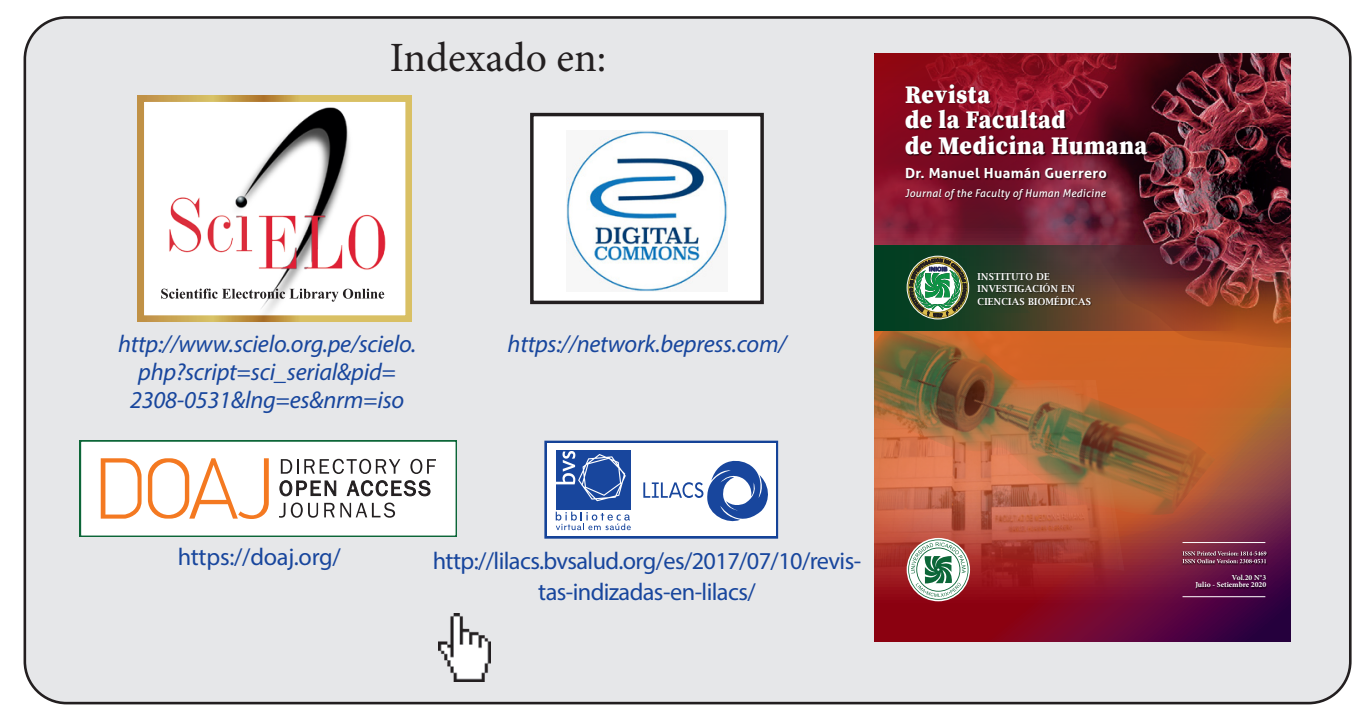

